\title{
Tangence
}

\section{Carnets d'automne (extraits)}

\section{Jean-Louis Roux}

Numéro 45, octobre 1994

Authenticité et littérature personnelle

URI : https://id.erudit.org/iderudit/025833ar

DOI : https://doi.org/10.7202/025833ar

Aller au sommaire du numéro

Éditeur(s)

Tangence

ISSN

0226-9554 (imprimé)

1710-0305 (numérique)

Découvrir la revue

Citer ce document

Roux, J.-L. (1994). Carnets d'automne (extraits). Tangence, (45), 147-159.

https://doi.org/10.7202/025833ar d'utilisation que vous pouvez consulter en ligne.

https://apropos.erudit.org/fr/usagers/politique-dutilisation/ 


\section{DOCWMini}

\section{Carnets d'automne (extraits) ${ }^{1}$}

\section{Jean-Louis Roux}

\section{Le jeudi 30 septembre 1993}

À la gare centrale, à cette même date en 1946 , je montais à bord d'un train en direction d'Halifax où je devais m'embarquer, à bord d'un cargo, et voguer vers l'Europe. J'emploie à dessein ce terme romantique, car cette traversée couronnait un rêve depuis trop longtemps caressé.

Comme tous les jeunes de ma génération, qui avaient eu le privilège de fréquenter les institutions d'enseignement jusqu'à leur baccalauréat, j'avais reçu une éducation centrée sur l'Europe et sur la culture française, plus particulièrement. J'avais vécu les années les plus importantes de mon évolution comme être humain, de 1939 à 1946, coupé de mes sources culturelles par un conflit qui ne me concernait pas - c'était du moins ma conviction, il y a cinquante ans -, un conflit que je rejetais instinctivement, aveuglément, que je détestais, dont je me refusais à reconnaitre les enjeux cruciaux, qui me frustrait des richesses inépuisables de cette vieille Europe déchirée, dont nos maitres et nos aînés nous avaient tracé une si séduisante image. Dans cet immense pays aux frontières exiguës, j'attendais impatiemment de pouvoir fuir ce qui me paraissait être l'atmosphère suffocante de ma famille, de mon milieu, de ma province, où je me voyais condamné à vie à la médiocrité. L'Europe, la France, Paris m'étaient d'autant plus attrayants qu'ils m'avaient été interdits, pendant près de six interminables années.

1 Ce document est une sélection d'extraits des Camets d'automne de JeanLouis Roux. Il est publié avec l'aimable autorisation de l'auteur. 
148

\section{$[\ldots]$}

Au début de mon séjour à Paris, les appartements vacants étaient réquisitionnés d'office, tant était sérieuse la crise du logement. Le peintre Julien Hébert en avait loué un, Cité des Fleurs près de la place Clichy, mais son départ de Montréal avait été retardé. Il m'avait fait demander par Jean de Rigault de lui rendre le service d'occuper les lieux, pour éviter qu'on ne s'en empare. J'acceptai avec plaisir: j'étais moi-même sans logis. La Cité, très vieillotte et mal entretenue, n'était pourtant pas désagréable. Une grille à chaque extrémité en interdisait le passage à la circulation automobile. Certains ministres du Second Empire y avaient, paraît-il, entretenu leurs maîtresses. C'est dans un de ces anciens nids d'amour, un peu sombre, un peu poussiéreux, que je m'installai. Luxe inusité, il y avait une salle de bain, attenante à la chambre à coucher.

\section{[...]}

Malgré ma détermination de ne jamais retourner au Canada, je n'avais toutefois pas rompu toutes les amarres avec mon pays natal. Ainsi, quelques mois après être débarqué à Calais, je participai à une série de conférences organisées par le fils du journaliste et éditeur Horace de Carbuccia, à ce moment-là condamné par contumace pour avoir soutenu le gouvernement de Vichy, durant l'occupation. Il devait du reste être plus tard acquitté de toute charge. L'événement fut présenté sous le nom d'Images $d u$ Canada. Parmi les conférenciers, Pierre Trudeau avait traité de politique fédérale et provinciale; Roger Rolland de littérature; Ludmilla Pitoëff y avait lu des poèmes d'Éloi de Grandmont. Pour ma part, j'avais décrit la vie quotidienne dans la métropole du Canada. Nous remportâmes un certain succès auprès d'un cercle restreint.

En une autre circonstance, tous les intellectuels et les artistes de la communauté canadienne de Paris avaient adressé aux journaux de Montréal une lettre de protestations au sujet de l'interdiction dont Maurice Duplessis avait frappé la projection du film Les enfants $d u$ paradis. Nous y décrivions notre honte de voir une conduite aussi ridicule et aussi mesquine faire la manchette des quotidiens de Paris. Parmi les signataires: Jean Gascon, le peintre Charles Daudelin et sa femme, Louise, le pianiste compositeur André Mathieu, Pierre Trudeau, Roger Rolland, Éloi de Grandmont, l'écrivain André Béland, etc. Cette lettre nous valut 
une semonce à l'Assemblée législative de Québec, sur le ton du paternalisme méprisant coutumier à cette époque, par la voix de René Chaloult, député indépendant, ultra-nationaliste, ancien directeur d'une publication à tendances racistes, L'action nationale.

\section{$[\ldots]$}

Cependant, m'installer à Paris définitivement, comme j'en avais eu l'intention en quittant Montréal, impliquait qu'il me fallait y travailler. Et d'abord obtenir un permis de travail; ce qui ne pouvait se faire, à moins d'avoir un contrat; mais on ne pouvait signer de contrat sans permis. Chinoiserie bureaucratique dont on venait à bout en trichant un peu. Mais, même muni d'un permis, je ne réussis jamais à travailler vraiment, sauf en compagnie de Ludmilla Pitoëff; et il est évident que cela présentait des limites.

Néanmoins, la première fois que je montai sur scène, dans le rôle de Krogstad de Maison de poupée, j'étouffais de bonheur, une fois le trac maitrisé. JOUER À PARIS, DEVANT UN PUBLIC PARISIEN! J'obtins même une assez bonne critique. Mauvais archiviste, je recherche en vain les coupures de journaux. Maison de poupée connut une reprise au Théâtre Gramont puis une très longue tournée, grâce à laquelle je visitai à peu près toute la France, un peu de la Belgique et de la Suisse et poussai une pointe jusqu'à Tunis.

Après Maison de poupée, je jouai, toujours avec Ludmilla Pitoëff, Le vray procès de Jeanne d'Arc, d'abord sur le parvis de la cathédrale de Rouen, puis sur la scène du théâtre SarahBernhardt. Mais ça ne démarrait pas.

Pas plus au cinéma, malgré toutes sortes de tentatives: visite chez Marc Allégret, qui devait tourner Maria Chapdelaine; lettre à Gide, pour solliciter un rôle dans La symphonie pastorale; audition devant Robert Bresson, pour le rôle du défroqué dans Le journal d'un curé de campagne; réponse à une petite annonce dans laquelle on disait rechercher des Canadiens blonds, grands et bilingues, pour le tournage du Grand cirque, d'après l'œuvre de l'as de l'aviation française, Pierre Closterman. Cette fois, la démarche réussit : nous reçûmes une convocation, Jean Gascon et moi. Je ne pus m'y rendre, mais Jean fut engagé. Dans un plan très large, à la condition d'être à l'affût, on peut le deviner courant en direction de son Hurricane, Canadien grand, blond et bilingue! 
150

Il fallut bien, avec le temps, me rendre à l'évidence et me décider à faire un choix. Valait-il mieux continuer à rêver, en trouvant probablement de petits emplois ici et là, et risquer une mort de somnambule? Ou ne fallait pas provoquer le réveil, fûtce brutalement, et prendre conscience de la réalité ? Je finis, malgré un profond chagrin, par admettre des choses auxquelles je me refusais, jusque-là : je n'appartenais pas à l'Europe, mais au Nouveau-Monde; je n'étais pas français mais canadien; ce n'était pas en France que je devais vivre et œuvrer, mais à Montréal. C'est là que je m'épanouirais, si j'avais à le faire; c'est là que je me rendrais le plus utile; c'est là que les meilleures chances me seraient offertes. La mort dans l'âme, je décidai donc de quitter Paris et tout ce que j'y aimais, en mars 1949.

J'eus pourtant un sursis. On allait tourner, aux studios de Billancourt, ce qui devait probablement être la première coproduction franco-québécoise: Le docteur Louise. Ti et J.-A. De Sève en étaient. En France, le producteur s'appelait l'abbé Vachon, un curé fort actif dans le mouvement d'action catholique. On cherchait évidemment des comédiennes et des comédiens canadiens, de préfêrence déjà à Paris, pour éviter des frais. Éloi de Grandmont, qui était rentré à Montréal, proposa mon nom qui fut agréé. Suzanne Avon, épouse d'un des Compagnons de la chanson, fut également engagée. Henri Poitras, lui, fit le voyage jusqu'en France. Le tournage fut prolongé, à cause d'un accident survenu à la vedette féminine, Madeleine Robinson. Je négociai un prolongement de contrat et le tout me rapporta finalement un peu plus de sept cents dollars. Pour moi et à l'époque, c'était une somme.

Je retardai mon départ au mois de juillet suivant et, pendant plusieurs semaines, j'entrepris une visite systématique de ce Paris qui allait tant me manquer. Pour pouvoir jouir entièrement de mon sursis, je décidai de vendre tout ce qui était monnayable de ce que je possédais, y compris mes vêtements. En rentrant rue Mollien, dépouillé de mes richesses, je trouvai un pneumatique: il fallait faire un raccord d'une scène dans laquelle je portais justement le costume bleu marine, dont je venais de me débarrasser. Je me précipitai chez le fripier. Trop tard: le costume gisait, en pièces détachées, sur un énorme tas de tissu. Je me rappelai alors que mon ami Wallace portait un costume semblable. J'allai le chercher à Rueil, le lendemain matin, et l'endossai au studio. La production n'y vit que du feu. 
Mes flâneries dans Paris, pendant ces dernières semaines, restent un de mes plus beaux souvenirs. Je disais adieu à tout: à mon Palais-Royal, à ma Place des Vosges, à mon Arc de triomphe du Caroussel, à mon Pré du Vert Galant, à ma place Furstenberg, à mon Pont-des-Arts, à ma Montagne Sainte-Geneviève... J'éprouvais de la nostalgie par anticipation, mais je m'imprégnais de la beauté d'une ville où partout triomphaient l'esprit, l'art, l'élégance et l'harmonie. Quel contraste avec ce que j'allais trouver.

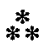

Je pris l'avion, un North Star de la Trans-Canada Airlines, le jeudi 21 juillet 1949. J'étais littéralement désespéré. Comme pour augmenter mon tourment, le vol se prolongea sur deux jours, avec escale de nuit à Londres et arrêt à Keflavik, base américaine en Islande, où nous fîmes un atterrissage en plein blizzard. J'étais assis à l'écart dans le sinistre aérogare, image de l'affliction, sans doute, car le commissaire de bord vint m'offrir deux bouteilles miniatures de J \& B, dans lesquelles je noyai une toute petite partie de mon chagrin.

J'atterris enfin à l'aéroport de Dorval, le samedi matin 23 juillet. Mes amis, parmi les meilleurs, m'ont rapporté que mes premières paroles avaient été pour leur déclarer que je n'étais "pas du tout content de les revoir!" Pour ma part, je n'ai souvenir que d'abattement, de fatigue et du vertige que me causait le bourdonnement incessant, à mes oreilles, des quatre moteurs Rolls Royce à hélices.

Il n'y avait qu'une solution: me plonger dans le travail. À la radio, on m'en offrait de partout. J'en étais ravi, car, après quelques jours à Montréal, ma secrète ambition était d'amasser rapidement le plus d'argent possible pour retourner à Paris. La laideur de la ville - et il est vrai qu'à cette époque Montréal n'était rien de plus qu'infect - me rebutait et j'étais hanté par les lumières et les rumeurs de Paris. Le soir, dans le salon de la maison paternelle que j'avais réintégrée, je me faisais tourner des disques de Piaf ou de Montand, des chansons de Prévert et Kosma et, la tête dans les mains, je sanglotais comme un petit garçon sans famille, que j'étais. "J'ai perdu ma maîtresse, sans l'avoir mérité..." 
152

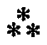

Côté théâtre, il y avait le projet d'Un fils à tuer, d'Éloi de Grandmont, sur lequel je travaillais déjà depuis quelques mois. J'étais à peine descendu de l'avion que nous fondions tous deux une compagnie, cette compagnie dont Jean Gascon et moi nous étions si souvent entretenus, lorsque j'allais déjeuner chez lui, le dimanche, rue de Birague, dans son petit appartement de bonnes, dont les fenêtres sous les combles donnaient sur la Place des Vosges. Il nous arrivait, après avoir ingurgité quelques verres de vin, de jouer les funambules et de passer de l'une à l'autre, par les toits, la place à nos pieds, six étages plus bas. Cette pauvre Mimi en avait des arrêts de cœur.

Ainsi naquit le Théâtre d'essai de Montréal, nom qui impliquait tout un projet. Dans le programme d'Un fils à tuer, j'expliquais que pour moi la vie n'était qu'une longue quête, jamais couronnée, de la perfection. De même pour le théâtre. J'allais donc faire mes modestes essais, conscient à l'avance de leurs défauts. Cette humilité cachait sans doute de grandes ambitions. Entre autres, celle de mettre sur pied un organisme dont j'offrirais la codirection à Jean Gascon, revint-il jamais à Montréal. Car, pour l'instant, Jean s'était joint au Centre dramatique de l'Ouest d'Hubert Gignoux, à Rennes, où il entamait une prometteuse carrière française.

La première de la pièce d'Éloi eut lieu le 4 octobre. Autour de moi, une forte distribution: Huguette Oligny, Ginette Letondal et Guy Provost. J'avais moi-même dessiné le décor. Exploit que je rééditerai pour la deuxième production, avant de m'apercevoir que je n'avais aucune disposition en la matière. Le sujet de la pièce reflétait nos préoccupations : un fils reprochait à ses parents d'avoir quitté la vieille Europe civilisée pour venir coloniser la Nouvelle-France. Au début, les parents ramenaient leur enfant fugueur qui s'était égaré en forêt. Le père le tenait aux épaules, la mère aux jambes. À la fin, le fils tentait de s'enfuir pour échapper à la sauvagerie d'un pays qu'il haïssait; mais, dans un geste de désespoir, le père l'abattait d'un coup de feu. Et la pièce se terminait sur le même tableau, les parents déposant sur la table le corps de leur pauvre enfant mal aimé. Un spectateur s'adressa alors à sa compagne, à haute et intelligible voix: “Viens-t'en: c'est ici qu'on est arrivé!" 
Faut-il le préciser: Un fils à tuer n'eut qu'un succès d'estime, comme on dit par euphémisme. Il est vrai qu'on adressa des reproches surtout à la pièce et qu'on épargna généralement la mise en scène et l'interprétation. Le mari de Ginette Letondal, le journaliste André Roche, s'occupait de notre publicité. Il eut l'idée d'organiser un petit sacndale, histoire de mousser l'événement: une rivalité amoureuse, entre Éloi et moi, qui devait aboutir à un duel à l'épée, derrière la prison de Bordeaux. Une photo fut même publiée, avec la complicité d'un hebdomadaire, Le petit journal. La police s'en mêla: pour l'agresseur - en l'occurrence Éloi de Grandmont à qui j'avais prétendument fauché sa bienaimée -, un duel constitue une tentative d'homicide. Nous fîmes l'objet d'une dépêche de la Presse canadienne et la photo de Monique Manuel, une jolie comédienne française qu'Éloi et moi étions censés nous disputer, fut publiée par Paris-Match. Tout le milieu, crédule ou sceptique, parlait de l'affaire. Il arrive qu'on en parle encore. Mais il n'y eut pas un spectateur de plus aux représentations de la pièce.

Nous n'avions pas l'abattage de Jean Desprez qui, malgré un éreintement unanime de la critique, parvint peu après à transformer en succès ce qui s'annonçait comme un four: sa Cathédrale, dans laquelle elle fit jouer une trentaine de comédiens et de comédiennes, appâtés par les rôles qu'elle leur offrait par ailleurs dans son feuilleton radiophonique, Jeunesse dorée. J'étais de la distribution: je jouais un homosexuel qui faisait des avances au jeune héros de la pièce, interprété par Roger Garceau et qui, pour toute réponse, en recevait une gifle. Avec sa raillerie irrésistible, Roger me disait, chaque fois que nous sortions de scène: "C'est bien parce que nous sommes au théâtre!"

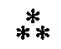

Ayant amassé une somme rondelette à force de feuilletons radiophoniques, je m'embarquai à New York, à bord de l'île-deFrance, le 19 novembre, de nouveau en route pour Paris. Coïncidence divertissante, j'avais assisté, la veille au soir, à une représentation de la pièce de Tennessee Williams, A Streetcar Named Desire, avec Huta Hagen et Anthony Quinn, qui n'étaient pas les créateurs des rôles de Blanche et de Kowalski, mais qui n'en 


\section{4}

étaient pas moins tous les deux admirables. Le soir de mon arrivée à Paris, le 25 novembre suivant, j'assistai à la même pièce, avec Arletty interprétant Blanche, dans une adaptation de Jean Cocteau. Une parodie, mes amis! Malgré son talent remarquable, Arletty, dès le début, me fit rire: son accent traînant de parigote était en effet du plus haut comique. Quant à Cocteau, il faisait déambuler sur scène de beaux jeunes Noirs à torses nus et noyait l'action dramatique dans une profusion de musique de jazz, New Orleans Style. Mon amour de Paris ne m'aveuglait pas au point de me faire avaler un tel navet.

Il y avait un peu plus de quatre mois que j'avais quitté Paris. Je le retrouvai avec énormément de joie, mais rien de comparable à ce que j'avais éprouvé, lors de ma première découverte. C'était une joie calme, sereine, douce, presque mélancolique. Un peu comme la joie de celui qui, après une longue absence, reverrait une femme toujours aimée, pour qui il aurait connu jadis une passion fulgurante. Cette fois, comprenais confusément que mon premier départ avait été un déchirement, une rupture, et que j'étais simplement revenu pour parvenir à ce que nous nous quittions, pour lors, comme des amis peuvent le faire, tendres et souriants.

Je repris, en attendant, le rythme de ma vie parisienne, faite de promenades, de visites de musées et d'assistance à des spectacles de toutes sortes. Une différence notoire: je me mis à écrire. J'avais bien commencé, environ deux ans plus tôt, une adaptation moderne de Lysistrata, d'Aristophane. Car mon propre cheminement, mes fréquentations et toute une série d'événements survenus au cours de l'année 1948 - l'assassinat de Gandhi, le suicide de Masaryk au moment de la prise du pouvoir des communistes à Prague, la guerre froide, l'arrestation de madame Joliot-Curie, lors d'une visite aux U.S.A., le manifeste des savants atomistes, etc. - avaient concouru à faire de l'avènement de la paix dans le monde une de mes préoccupations majeures. Mais après quelques semaines de travail et une première scène à peine ébauchée, j'avais abandonné le projet.

Pourtant, deux spectacles firent cheminer, en mon esprit, l'idée d'écrire une pièce. D'abord L'ombre d'un franc-tireur, d'O'Casey, puis Deirdre des douleurs, de Yeats. Deux auteurs irlandais. À la suite de quelques lectures, après avoir assisté à ces deux spectacles, me frappèrent plusieurs similitudes entre notre 
situation sociale, politique et culturelle, au Québec, et celle des Irlandais. Deirdre, inspirée d'une légende irlandaise, m'amena à consulter notre propre folklore. Me revinrent en mémoire plusieurs des contes des anciens Canadiens, qui faisaient partie de la petite bibliothèque de mon père et que j'avais lus et relus, à l'âge de dix ou douze ans: La Corriveau, La tête à Pitre, La chassegalerie, etc. Je m'arrêtai à celui de Rose Latulippe. Histoire de laisser errer mon imagination, je tins à ne pas le relire immédiatement. De toute façon, à Paris, c'eût été difficile.

Le personnage de Rose certes m'inspirait; mais j'étais davantage fasciné par celui du diable. J'essayais de me figurer ce qui, en lui, avait séduit la jeune fille. Je commençai donc par écrire une des dernières scènes de la pièce: celle où Rose valse avec son cavalier diabolique, jusqu'à en perdre le souffle et la vie, emportée dans les flammes de la passion.

Après trois mois, j'avais pratiquement terminé cette scène de quelques pages, mais j'avais également épuisé mes ressources pécuniaires, mes recherches de travail s'étant révélées encore plus infructueuses qu'à mon premier séjour. D'autre part, mes liens affectifs s'étaient relâchés, sans que j'en éprouve de désespoir. Pour lors, beaucoup plus serein qu'au mois de juillet précédent, je me soumis à mon sort. Il me fallait rentrer et, cette fois, de façon définitive. Nous étions en mars 1950.

\section{***}

Une fois à Montréal, je repris mon gagne-pain à la radio et je jouai aussi quelques rôles sur scène dans La folle de Chaillot et dans Pygmalion, pour le M.R.T. français, ainsi que dans L'Arlésienne, pour les Festivals de Montréal. Mais je renouai surtout avec ma Rose et son irrésistible cavalier. À l'automne, j'avais terminé ma pièce, dont la première eut lieu, toujours au Gesù, le 20 février 1951.

Comme pour la pièce d'Éloi de Grandmont, j'avais réuni une belle distribution: Thérèse Cadorette, unique interprète féminine, entourée de Gilles Pelletier, Roger Garceau, Jean Duceppe, JeanLouis Paris, Jean-Pierre Masson, Robert Gadouas et Jean Coutu, le séduisant prince des ténèbres. Assez révélateur du style de la pièce, les personnages portaient le nom de leur emploi: Leriche, Lenoble, 
Lebeau, Loisif et Lamoureux, que jincarnais. Assez révélateur aussi de son intérêt, la pièce tomba dans l'oubli après les quelques représentations qu'elle connut alors, malgré les éloges dont me gratifia le poète Alain Grandbois dans le programme de la soirée.

La critique fut assez sévère pour l'auteur débutant que j'étais. Celle du quotidien Le Canada blessa ma vanité à un tel point qu'un midi, j'en giflai le signataire, Roland Côté, au bar du restaurant $L e 400$, lieu de rendez-vous de la communauté théâtrale. Je n'avais que vingt-huit ans et j'étais encore d'un tempérament insoumis. L'après-midi, je reçus un coup de fil de Jean Coutu, m'avisant que l'offensé entamait des procédures judiciaires contre moi. Je connaissais un avocat, Roger Nadeau, chroniqueur d'occasion au Service international de Radio-Canada, où je travaillais, avec René Lévesque entre autres, comme speaker et rédacteur. Je lui passai un coup de fil et le priai de me représenter dans cette querelle. Il me répondit qu'en toute autre occasion il serait ravi de défendre mes intérêts mais qu'en l'occurrence, il se trouvait être le procureur de son neveu, Roland Côté! L'affaire se régla hors cour, par une poignée de mains.

Le Théâtre d'essai de Montréal comptait donc dans son répertoire deux créations d'auteurs canadiens en deux productions. Estimable bilan artistique. Le bilan financier n'était pas aussi brillant. Après un peu plus d'un an et demi d'existence, il comportait un déficit de près de deux mille cinq cents dollars. J'assumai la dette et grâce à un prêt sans intérêts que me consentit ma belle-mère, la journaliste Odette Oligny, je pus payer immédiatement toutes les factures, y compris les cachets des comédiens.

Au printemps, je reçus la nouvelle du retour de Jean Gascon. Non que son travail se soit interrompu au Centre dramatique de l'Ouest; mais la naissance prochaine d'un deuxième enfant laissait entrevoir une vie extrêmement pénible, pour lui et sa femme Mimi, dans une France où les conditions matérielles n'étaient pas encore revenues à leur niveau normal. Je savais que la décision devait comporter un grand sacrifice pour Jean, puisque son travail à Rennes pouvait lui offrir la voie d'un bel avenir. Mais j'étais heureux, en bon égoïste, à la perspective de retrouver un 
compagnon avec qui j'avais partagé mes premières expériences théâtrales, au Collège Sainte-Marie, chez les Compagnons de Saint-Laurent et dans la Compagnie Ludmilla Pitoëff, ainsi que ma décision d'abandonner la médecine pour embrasser la carrière de comédien. À l'université, en effet, nous avions passé un pacte dont les termes stipulaient que si l'un de nous deux faisait le grand saut, l'autre devait suivre. C'est ce qui se produisit. Jean, le premier, et moi, l'instant d'après.

Nous nous sommes retrouvés presque un an, jour pour jour, après mon deuxième départ de Paris. Et nous avons immédiatement repris nos discussions là où nous les avions laissées, lors d'une visite que je lui avais faite en Bretagne, au sujet de la création d'une compagnie de théâtre. Se joignirent à nous, à Montréal, Éloi de Grandmont et André Gascon. Je discernai toutefois un changement d'attitude chez Jean: il n'était plus question de partager la direction d'une éventuelle troupe. Fort de son expérience et de ses succès avec la Compagnie Grenier-Hussenot, de Paris, et avec Hubert Gignoux, au Centre dramatique de l'Ouest, Jean Gascon était déterminé à assumer seul les responsabilités artistiques de l'organisme que nous voulions fonder. Je dus me rendre à ses arguments; mais à l'époque, son attitude me chagrina. Le temps et la pratique du métier m'apprirent pourtant que Jean avait eu raison: les directions multicéphales, surtout au thêâtre, fonctionnent généralement mal et font long feu.

Toutefois, la fondation de notre compagnie faillit bien ne jamais avoir lieu. Le père Legault, prévoyant la fin des activités des Compagnons de Saint-Laurent, en offrit la direction à Jean Gascon. Les pourparlers furent rompus après une entrevue "au sommet "à laquelle j'assistai, dans le secrétariat des Compagnons, rue Sherbrooke à l'angle de la rue Delorimier. Nous exigions le départ pur et simple de l'ancien directeur des Compagnons, alors qu'il désirait, lui, conserver un poste mal défini, un peu conseiller, un peu patriarche, un peu éminence grise. Ce lien avec le passé et le danger constant d'intrusion du fondateur des Compagnons d'hier dans la gestion et l'organisation de ceux de demain étaient, à nos yeux, totalement inadmissibles. Nous nous sommes quittés, un peu mélancoliques, personne n'ayant songé - était-ce symbolique? à faire de la lumière dans la pièce où nous nous trouvions.

Par ailleurs, nous avions eu de nombreuses entrevues avec un avocat de Québec, Mark Drouin, que nous avait fait connaître 
son cousin, le comédien Denis Drouin. Le prénom "Mark" s'orthographiait avec un $k$, selon une mode courante, à l'époque, dans la ville de Québec. Il avait du reste un frère qui se prénommait Ross. Mark, un peu blasé par le monde politique où il baignait, était fasciné par notre enthousiasme de néophytes et je le dis avec sympathie - émoustillé à l'idée de fréquenter un milieu d'artistes. Il répétait qu'il allait se cultiver "par osmose." Avec un groupe d'amis, il nous garantit un emprunt de cinq mille dollars à la banque: c'était suffisant pour lancer une première saison.

Nous eûmes une réunion spéciale pour décider du nom de notre compagnie. C'est Éloi de Grandmont qui emporta facilement l'adhésion unanime en proposant Théâtre du Nouveau Monde. À la fois un défi et un programme. Lors d'une autre réunion, nous discutâmes, en présence de Mark Drouin, des structures juridiques de l'organisme. Mark nous conseilla une société par actions: il s'occuperait de la paperasse admninistrative, une fois réparties ces actions entre nous. Outre Jean, Mark et moi, il y avait André Gascon, Êloi de Grandmont, Georges Groulx, Guy Hoffman, Charles Lussier, Robert Gadouas et Émile Caouette. D'emblée, Jean exigea de détenir un bloc d'actions plus important que tout autre. Derechef, cette attitude ma chagrina, mais je me contentai de protester sans vigueur. C'est, je crois, un trait de mon caractère: je suis prêt à rompre en visière pour défendre des principes mais, quant à mes intérêts personnels, je me montre volontiers indolent. Et puis, je ne voulais pas compromettre, par un désaccord au sujet d'actions qui ne portaient qu'une valeur nominale, l'avenir de notre compagnie. Il en fut fait selon le désir de Jean. Je détenais le deuxième bloc d'actions le plus important; mais à eux deux, Jean et son frère André étaient majoritaires. Plus tard, cela se révéla d'une importance capitale pour moi.

Mais, pour lors, c'est dans l'euphorie que fut convoquée une conférence de presse, le 6 juillet 1951, sur la scène du Gesù. Il y avait cohue. Jean Gascon fit lecture d'un communiqué. Fernand Seguin, qui l'avait traduit, fit de même en anglais. Car nous entendions attirer également le public anglophone de Montréal. Les termes en étaient extrêmement sobres. On insistait sur le sérieux de l'entreprise, du point de vue administratif autant que du point de vue artistique. De manifeste, aucun. Rien sur notre position esthétique. Rien sur notre rôle social. Nous ne faisions que décla- 
rer que le répertoire serait choisi sous le signe de l'éclectisme. Sans doute voulions-nous signifier par là qu'aucun autre critère ne nous guiderait que nos goûts personnels: j'aime ou je n'aime pas; j’aimerais monter telle pièce, j'aimerais jouer tel rôle. En réalité, nous fondions un théâtre parce que nous aimions être sur scène et que nous étions viscéralement convaincus de ne pouvoir nous en passer. Opération de survie: nous pensions d'abord à nous; le public ne venait qu'en second lieu. Ce n'est pas un reproche; d'ailleurs, si reproche il y avait, je devrais me l'adresser à moi aussi bien qu'aux autres. Non: c'est un simple constat. Finalement, faire du théâtre parce qu'on aime ça constitue une des meilleures motivations qui soient.

Par la même occasion, les directeurs du Théâtre du Nouveau Monde annonçaient le lever de rideau initial, le 9 octobre suivant, à $21 \mathrm{~h}$, sur L'avare, de Molière. 